\title{
Erratum to: Validation of the BRCA1 antibody MS110 and the utility of BRCA1 as a patient selection biomarker in immunohistochemical analysis of breast and ovarian tumours
}

\author{
Roy Milner • Helen Wombwell • Sonia Eckersley • \\ Donna Barnes • Juli Warwicker • Erica Van Dorp • \\ Rachel Rowlinson • Simon Dearden • Glen Hughes • \\ Chris Harbron • Bob Wellings • Darren Hodgson • \\ Chris Womack • Neil Gray • Alan Lau • Mark J. O'Connor • \\ Catherine Marsden • Alexander J. Kvist
}

Published online: 19 April 2013

(C) Springer-Verlag Berlin Heidelberg 2013

Erratum to: Virchows Arch (2013) 462:269-279 DOI 10.1007/s00428-012-1368-y

The original version of this article, unfortunately, contained an error.

Rachel Rowlinson, who also contributed to this paper, was inadvertently ommitted in the author group in the original version.

She is now listed in this article.

The online version of the original article can be found at http://dx.doi.org/ 10.1007/s00428-012-1368-y.

R. Milner $\cdot$ H. Wombwell $\cdot$ S. Eckersley $\cdot$ D. Barnes

J. Warwicker $\cdot$ E. Van Dorp $\cdot$ R. Rowlinson $\cdot$ S. Dearden •

G. Hughes $\cdot$ C. Harbron $\cdot$ B. Wellings $\cdot$ D. Hodgson $\cdot$

C. Womack · N. Gray $\cdot$ A. Lau $\cdot$ M. J. O'Connor · C. Marsden •

A. J. Kvist

AstraZeneca, Alderley Park,

Macclesfield, Cheshire, UK

A. J. Kvist $(\square)$

19 F7 Mereside, Alderley Park,

Macclesfield, SK10 4TG Cheshire, England, UK

e-mail: Alexander.kvist@astrazeneca.com 